\title{
Recurrent borderline phyllodes tumor in nipple: a rare case report and review of the literature
}

\author{
Denghua Sun", Lu Tang", Hua Xing, Lijuan Zhang, Le Zhang \\ Department of Breast Surgery, China-Japan Union Hospital, Jilin University, Changchun 130033, China \\ \#These authors contributed equally to this work. \\ Correspondence to: Dr. Lijuan Zhang; Dr. Le Zhang. Department of Breast Surgery, China-Japan Union Hospital, Jilin University, 126 Xiantai Street, \\ Changchun 130033, China. Email: Ljzhang1985@163.com; Lezhangmed@163.com.
}

\begin{abstract}
Phyllodes tumor (PT) of the breast is a rare tumor. They are usually located in the four quadrants of the mammary gland and may also appear below the nipple. Although there have been a number of cases reported, here we report a very rare case of this tumor in an unusual location. The patient has been diagnosed with right breast borderline PT, who accepted breast-conserving surgery. Twelve months after surgery we found right nipple enlargement, without nipple discharge, ipsilateral and contralateral breast without palpable mass. Interestingly, ultrasound showed a lesion in the right nipple: a hypoechoic mass with a diameter of $2.0 \mathrm{~cm}$ was seen in the nipple, with no significant calcification. The patient underwent nipple-sparing mastectomy without postoperative adjuvant radiotherapy. Histopathological and immunohistochemical analysis demonstrated a borderline PT. There were no signs of recurrence after two years of follow-up. We consider that wide surgical resection and negative margin are still the main methods for the treatment of recurrent borderline PTs.
\end{abstract}

Keywords: Breast; phyllodes tumor (PT); nipple; recurrent; case report

Submitted Oct 21, 2019. Accepted for publication Jan 04, 2020.

doi: $10.21037 /$ gs.2020.01.17

View this article at: http://dx.doi.org/10.21037/gs.2020.01.17

\section{Introduction}

Phyllodes tumor (PT) is a rare breast tumor that accounts for only $0.3 \%$ to $0.9 \%$ of all primary breast tumors, and occurs in 35-55 year old women $(1,2)$. In 2003, the World Health Organization (WHO) named it PT. The histological features of PT can be divided into benign (grade I), borderline (grade II) and malignant (grade III) categories (3). PTs can occur in all parts of the mammary gland, including upper outer, lower outer, upper inner, lower inner, and central of breast $(4,5)$. However, PTs growing in the nipples have not been reported so far.

In this report, we presented the clinical features of one case of borderline PT recurrence in the nipple, in order to improve the diagnosis and treatment of this kind of tumor. We present the following case in accordance with the
CARE Guideline (6).

\section{Case presentation}

A 32-year-old female patient with no smoking or alcohol consumption history presented with a right breast mass in October 2015. The distance between the tumor and the nipple was about $2.0 \mathrm{~cm}$. There was no pain or sensory complaints related to this tumor. No nipple discharge and no masses were identified in either breast. An ultrasound examination confirmed a regular hypoechoic solid mass in the right breast measuring $3.0 \mathrm{~cm}$ in longest diameter. During resection of right breast mass under general anesthesia, intraoperative pathological result revealed borderline PT of breast. Then we chose breast-conserving surgery, and intraoperative frozen section analysis was 

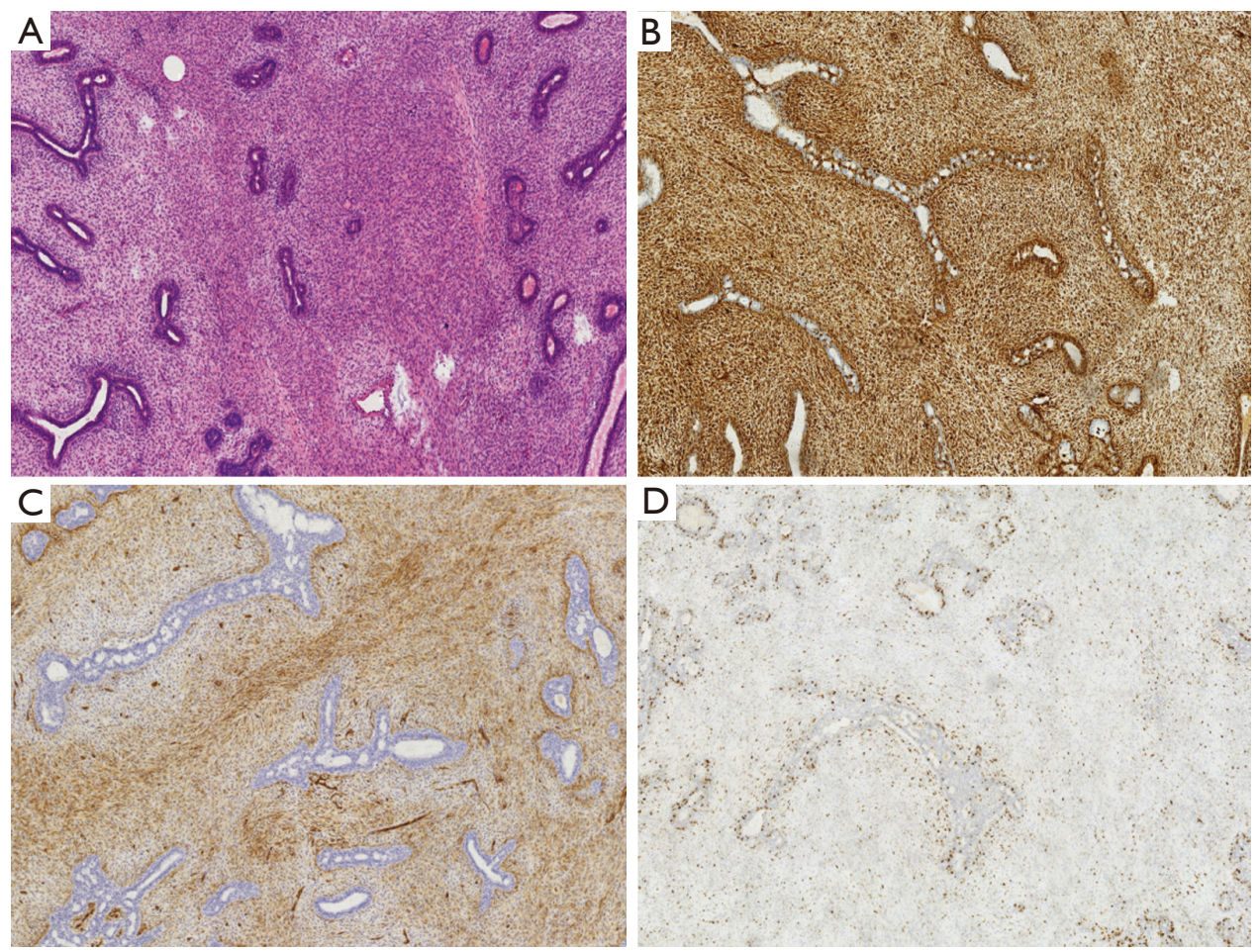

Figure 1 Image of paraffin histologic pathology diagnosis and IHC staining of first surgery $(\times 100)$. (A) Borderline phyllodes tumor can be seen in the tissue; (B) Vimentin positive expression can be detected by IHC staining; (C) CD34 positive expression can be detected by IHC staining; (D) Ki-67 positive expression can be detected by IHC staining.

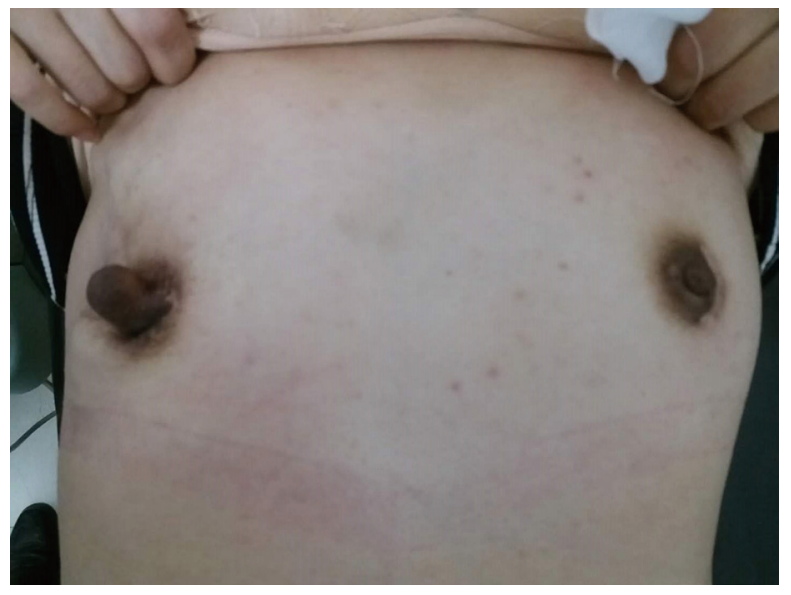

Figure 2 Preoperative nipple photo of patient. Before surgery, the enlarged nipple on the right breast was visibly prominent.

performed on the margin of the four directions around the tumor and the posterior margin of the nipple-areola complex. All excision margins were larger than $1 \mathrm{~cm}$. The pathological results of the incision margin were negative, and the operation was immediately terminated. Paraffin pathological report showed the same result (Figure 1A). Immunohistochemistry of the tissue was positive for Vimentin, CD34, and Ki-67 (Figure 1B,C,D). The patient was discharged without any additional treatment.

One year later, the patient discovered a swelling in the right nipple. Physical examination identified a nonmobile mass in the right nipple, measuring $2.0 \mathrm{~cm}$ in diameter (Figure 2), and no clear mass was found in the rest of the breast; other superficial lymph nodes were not significantly enlarged. Ultrasound examination revealed a regular hypoechoic masses $(2.24 \mathrm{~cm} \times 0.96 \mathrm{~cm})$ in the right nipple (Figure 3). The mass in right nipple was resected under general anesthesia (Figure 4). The intraoperative pathological result accorded with PT. We confirmed the recurrence of PTs based on pathological findings. Then we continued to perform nipple-sparing mastectomy without immediate breast reconstruction, and also performed intraoperative frozen pathological examination of the margin of posterior nipple-areola complex. The pathological result of the incision margin was negative. 
Histopathological examination confirmed a borderline PT of the breast (Figure $5 A$ ). Immunohistochemistry of the tissue was positive for Vimentin, CD34, and Ki-67 (Figure $5 B, C, D)$. The patient was discharged home and refused to receive adjuvant radiotherapy. Two years later, she remains disease free with no signs of recurrence (Figure 6).

\section{Discussion}

PT of the breast is a rare disease, which belongs to the fibrous epithelial tumor. It is almost benign, but the recurrence rate is high. A few patients will experience hematogenous dissemination with distant metastasis. However its origin is unclear, some believe that it not only originates from primitive mesenchymal cells, but also from fibroblasts and myofibroblasts. The proliferation and differentiation of stromal cells, to a large extent, reflects

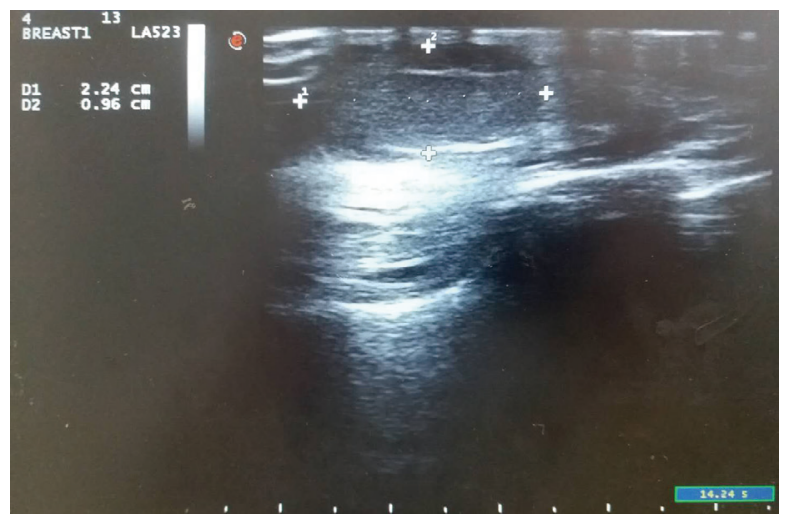

Figure 3 Ultrasound of the tumor in the nipple. A low echo mass of $2.24 \mathrm{~cm} \times 0.96 \mathrm{~cm}$ can be seen in the nipple. The boundary is still clear and the shape is regular. benign or malignant tumors and determines the biological behavior of the tumor (7). The incidence of PT of the breast is low, the vast majority of which occur in women. It is more common in premenopausal women, with an average age of onset of about 40 years old (8). At present, the etiology of this disease is unclear. Besides race and age, other factors may include fertility, lactation, endocrine, and metabolic changes. Some researchers believe that PTs are associated with fibroids. Breast fibroadenoma has been reported in $12-20 \%$ of patients, and $21 \%$ of patients with PTs experience simultaneous fibroadenoma, but whether all PTs originate from fibroadenomas remains controversial (9).

Clinically, PTs can be divided into three groups, including benign, malignant and borderline (3). The degree of deterioration of benign PTs is low and the growth rate is generally long. However, the blood supply of malignant tumors and borderline tumors is more abundant, which may be a reason for the continued growth of such tumors.

The disease is mainly characterized by painless solitary lump, usually located in the upper quadrant of the mammary gland, and there is no obvious difference in the incidence of mammary gland between the left and right sides. The mass generally does not invade the pectoral muscles and skin, which the boundary is clear. If the mass is larger, the surface of the skin becomes thin and light due to increased tension and the emergence of varices. Severe cases may be rare due to the rupture of blood and nipple retraction. Approximately $20 \%$ of patients present with large axillary lymph nodes that are likely reactive to infection and tumor necrosis. True lymph nodal metastasis occurs in less than $1 \%(10)$. The main mode of metastatis of PT is hematogenous occurring in $3-24 \%(8,10)$, typically to the lung, bone, and liver. Core needle biopsy has a good diagnostic sensitivity. There appears to be a statistically
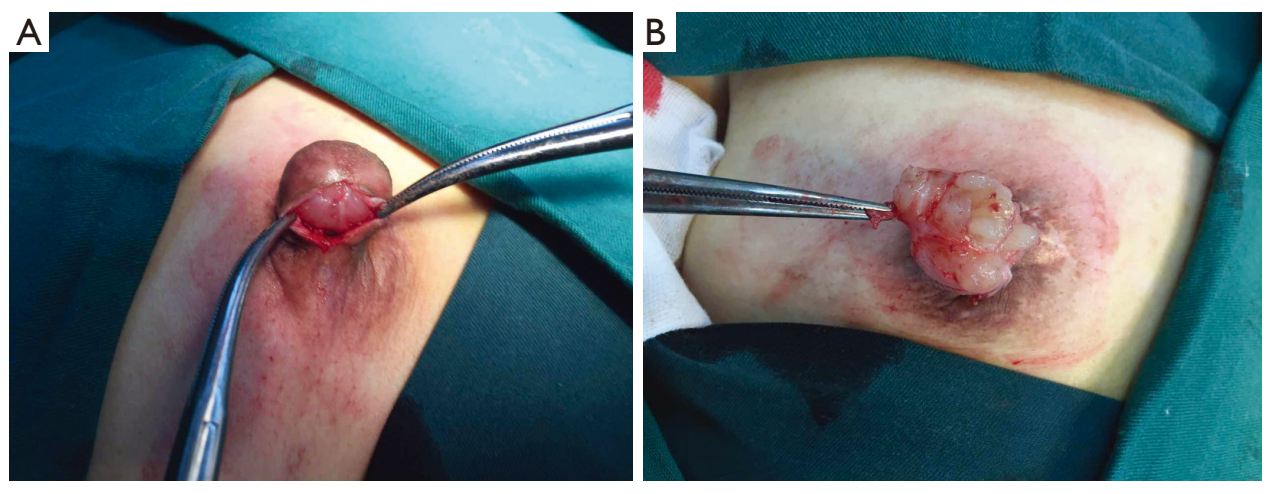

Figure 4 Intraoperative photo of the mass. The tumor was embedded in the nipple, which was removed completely. 

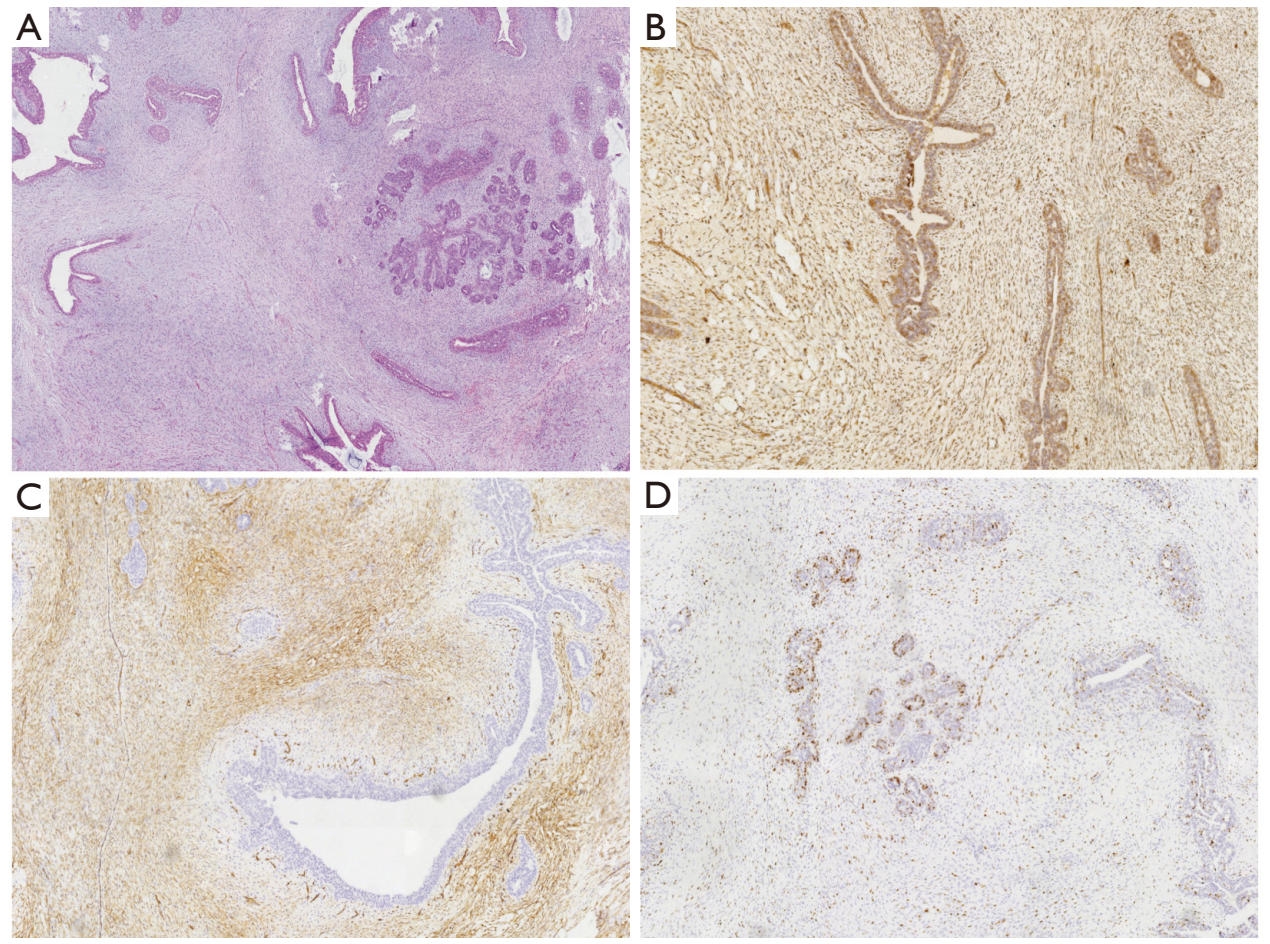

Figure 5 Image of paraffin histologic pathology diagnosis and IHC staining of secondary surgery $(\times 100)$. (A) Borderline phyllodes tumor can be seen in the tissue; (B) Vimentin positive expression can be detected by IHC staining; (C) CD34 positive expression can be detected by IHC staining; (D) Ki-67 positive expression can be detected by IHC staining.

significant association between the histological grade of PT and tumor size, radiological suspicion, mitotic count, cellular atypia, stromal cellularity, and tumor necrosis (3).

Wide excision with clear margin is the most important treatment for this disease (11). The choice of the first surgical procedure is closely related to the recurrence rate. Negative margin is to reduce the independent prognostic factors for local recurrence rate and prolong the survival period, which is independent of the tumor size and histological grade (12-17). So the principle of treatment is local extensive resection of PT underwent resection, in order to ensure the negative surgical margin. The current consensus is that a wide range of surgical procedures should achieve as many as $1-2 \mathrm{~cm}$ negative margins $(18,19)$. Mastectomy was indicated for larger tumors, recurrent tumors, and malignant PT $(4,16)$. However, in this case, the recurrence occurred within 2 years after the first operation. Interestingly, the location of recurrence was in the nipple which was very rare indeed. We took nipplesparing mastectomy which was considered as wide excision with negative margin during the second operation. We considered that the main cause of recurrence may be related to mitoses, tumor border, tumor necrosis, stromal cellularity, stromal atypia and notably stromal overgrowth (16). And the distance between the tumor and the nipple before first surgery was also a significant risk factor, although the pathological results of posterior margin of the nipple-areola complex was negative.

In recent years, a series of studies to explore the risk factors of recurrent breast PT, in addition to histological grade, putative prognostic factors including young age ( $<35$ years), tumor necrosis and positive surgical margins. Recent reports recommend adjuvant treatment of borderline cases with more than $5 \mathrm{~cm}$ in diameter, with histological evidence of stromal overgrowth as these tumors appear to have greater metastatic potential $(20,21)$. There was report in the literature that malignant PTs were treated with adjuvant radiotherapy, however the patient's benefit was uncertain (22). Varghese $e t$ al. revealed that Surgical resection with adequate margins $(>1 \mathrm{~cm})$ gave excellent local control in benign and borderline tumors (23). For patients with borderline 


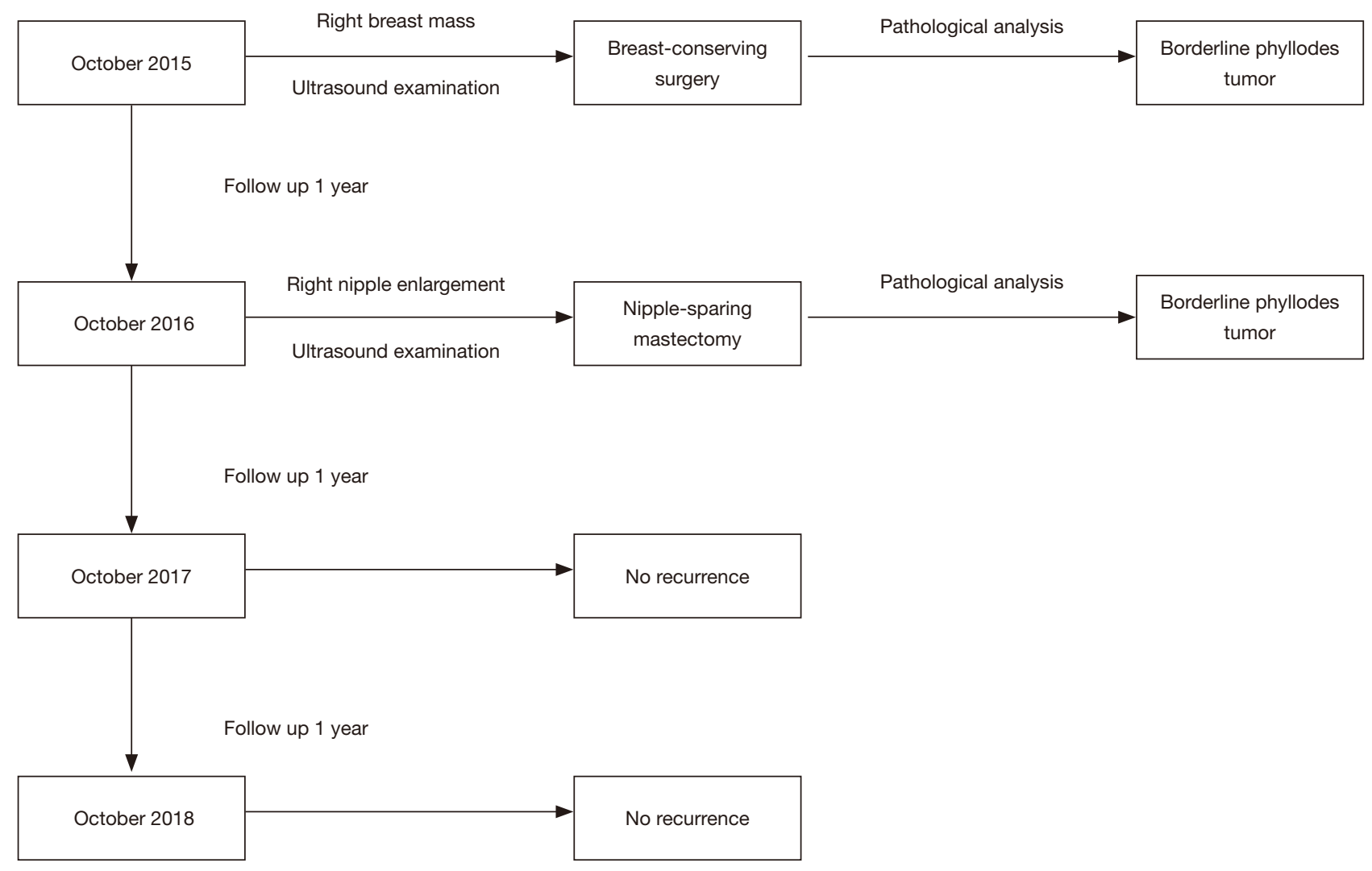

Figure 6 Timeline image of patient's history. A timeline can present relevant events in the patient's history in chronological order in a figure.

PT, local radiotherapy was useful, if margins were close or positive even after the best surgical resection (23). Yogi et al. also reported adjuvant radiotherapy was appropriate treatment for aggressive borderline and malignant PT (24). Choi et al. believed that patients with large malignant PT $(\geq 5 \mathrm{~cm})$ also had a higher risk of local recurrence who received margin-negative breast conserving surgery. Therefore, additional local treatment should be considered for such patients (17). Zeng et al. discovered that adjuvant radiotherapy was not superior to no radiotherapy in the total mastectomy group. And adjuvant radiotherapy for borderline and malignant PT decrease the local recurrence rate in patients undergoing breast conserving surgery (25). In this case, the patient refused to receive post-operative adjuvant radiotherapy. During the followup period of 2 years after the second operation, no signs of local recurrence were found. Therefore, more work is required to establish if radiotherapy is a beneficial treatment for this type of PT. Of course, we will further extend the follow-up time to verify. Molecular markers examined have now included epidermal growth factor receptor (EGFR), vascular endothelial growth factor (VEGF), beta-catenin, c-kit and Wnt signaling pathway, and tumor cell glycolysis (26-28). Thus far, however, there are no known effective targeted therapies for this tumor.

Our case report also has its limitation. Breast reconstruction, including breast prosthetic implant or autologous tissue reconstruction, can be performed immediately after nipple-sparing mastectomy. In this way, the shape of the breast can be restored to the greatest extent. In this case report, the patient refused to accept reconstruction, so we were unable to repair the appearance.

\section{Patient perspective}

The tumor recurred after my first operation, which worried me a lot, but fortunately the second operation was successful. I didn't agree to continue the radiotherapy after 
the second operation. I think it is possible to monitor the recurrence through regular postoperative review.

\section{Conclusions}

We presented a rare case of recurrent borderline PT in the nipple. Wide surgical excision with negative margins remains the mainstay of therapy for this unusual tumor.

\section{Acknowledgments}

Funding: This study was supported by Wu Jieping Medical Foundation (320.6750.18120).

\section{Footnote}

Conflicts of Interest: All authors have completed the ICMJE uniform disclosure form (available at http://dx.doi. org/10.21037/gs.2020.01.17). The authors have no conflicts of interest to declare.

Ethical Statement: The authors are accountable for all aspects of the work in ensuring that questions related to the accuracy or integrity of any part of the work are appropriately investigated and resolved. Our study was approved by the ethics committee of China Japan Union Hospital of Jilin University. Written informed consent was obtained from the patient for publication of this Case report and any accompanying images.

Open Access Statement: This is an Open Access article distributed in accordance with the Creative Commons Attribution-NonCommercial-NoDerivs 4.0 International License (CC BY-NC-ND 4.0), which permits the noncommercial replication and distribution of the article with the strict proviso that no changes or edits are made and the original work is properly cited (including links to both the formal publication through the relevant DOI and the license). See: https://creativecommons.org/licenses/by-nc-nd/4.0/.

\section{References}

1. Cheng F, Qin JJ, Yu MN, et al. De novo phyllodes tumor in an adolescent female after liver transplantation. Pediatr Transplant 2011;15:E12-4.

2. Testori A, Meroni S, Errico V, et al. Huge malignant phyllodes breast tumor: a real entity in a new era of early breast cancer. World J Surg Oncol 2015;13:81.
3. Efared B. Phyllodes tumors of the breast: clinicopathological analysis of 106 cases from a single institution. Breast Dis 2018;37:139-45.

4. Wang H, Wang X, Wang CF. Comparison of clinical characteristics between benign borderline and malignant phyllodes tumors of the breast. Asian Pac J Cancer Prev 2014;15:10791-5.

5. Chen WH, Cheng SP, Tzen CY, et al. Surgical treatment of phyllodes tumors of the breast: retrospective review of 172 cases. J Surg Oncol 2005;91:185-94.

6. Riley DS, Barber MS, Kienle GS, et al. CARE guidelines for case reports: explanation and elaboration document. J Clin Epidemiol 2017;89:218-35.

7. Grenier J, Delbaldo C, Zelek L, et al. [Phyllodes tumors and breast sarcomas: a review]. Bull Cancer 2010;97:1197-207.

8. Mitus JW, Blecharz P, Reinfuss $M$, et al. Changes in the clinical characteristics, treatment options, and therapy outcomes in patients with phyllodes tumor of the breast during 55 years of experience. Med Sci Monit 2013;19:1183-7.

9. Wang ZC, Buraimoh A, Iglehart JD, et al. Genome-wide analysis for loss of heterozygosity in primary and recurrent phyllodes tumor and fibroadenoma of breast using single nucleotide polymorphism arrays. Breast Cancer Res Treat 2006;97:301-9.

10. Spitaleri G, Toesca A, Botteri E, et al. Breast phyllodes tumor: a review of literature and a single center retrospective series analysis. Crit Rev Oncol Hematol 2013;88:427-36.

11. Mitus J, Reinfuss M, Mitus JW, et al. Malignant phyllodes tumor of the breast: treatment and prognosis. Breast J 2014;20:639-44.

12. Asoglu O, Ugurlu MM, Blanchard K, et al. Risk factors for recurrence and death after primary surgical treatment of malignant phyllodes tumors. Ann Surg Oncol 2004;11:1011-7.

13. Tan PH, Thike AA, Tan WJ, et al. Predicting clinical behaviour of breast phyllodes tumours: a nomogram based on histological criteria and surgical margins. J Clin Pathol 2012;65:69-76.

14. J. Barth R J. Margin negative, breast conserving resection: adequate for benign phyllodes tumors, but inadequate therapy for borderline and malignant phyllodes tumors. Breast Cancer Res Treat 2013;142:463-4.

15. Kim S, Kim JY, Kim DH, et al. Analysis of phyllodes tumor recurrence according to the histologic grade. Breast Cancer Res Treat 2013;141:353-63. 
16. Lu Y, Chen Y, Zhu L, et al. Local Recurrence of Benign, Borderline, and Malignant Phyllodes Tumors of the Breast: A Systematic Review and Meta-analysis. Ann Surg Oncol 2019;26:1263-75.

17. Choi N, Kim K, Shin KH, et al. Malignant and borderline phyllodes tumors of the breast: a multicenter study of 362 patients (KROG 16-08). Breast Cancer Res Treat 2018;171:335-44.

18. Kapiris I, Nasiri N, A'Hern R, et al. Outcome and predictive factors of local recurrence and distant metastases following primary surgical treatment of high-grade malignant phyllodes tumours of the breast. Eur J Surg Oncol 2001;27:723-30.

19. Tan BY, Acs G, Apple SK, et al. Phyllodes tumours of the breast: a consensus review. Histopathology 2016;68:5-21.

20. Chaney AW, Pollack A, McNeese MD, et al. Primary treatment of cystosarcoma phyllodes of the breast. Cancer 2000;89:1502-11.

21. Ossa CA, Herazo F, Gil M, et al. Phyllodes tumor of the breast: a clinic-pathologic study of 77 cases in a Hispanic cohort. Colomb Med (Cali) 2015;46:104-8.

22. Macdonald OK, Lee CM, Tward JD, et al. Malignant phyllodes tumor of the female breast: association of primary therapy with cause-specific survival from the

Cite this article as: Sun D, Tang L, Xing H, Zhang L, Zhang L. Recurrent borderline phyllodes tumor in nipple: a rare case report and review of the literature. Gland Surg 2020;9(2):452-458. doi: 10.21037 /gs.2020.01.17
Surveillance, Epidemiology, and End Results (SEER) program. Cancer 2006;107:2127-33.

23. Varghese SS, Sasidharan B, Manipadam MT, et al. Radiotherapy in Phyllodes Tumour. J Clin Diagn Res 2017;11:XC01-3.

24. Yogi V, Singh OP, Malviya A, et al. Effect of postoperative time for adjuvant radiotherapy in malignant phyllodes tumor: An institutional experience. J Cancer Res Ther 2018;14:1054-8.

25. Zeng S, Zhang X, Yang D, et al. Effects of adjuvant radiotherapy on borderline and malignant phyllodes tumors: A systematic review and meta-analysis. Mol Clin Oncol 2015;3:663-71.

26. Ho SK, Thike AA, Cheok PY, et al. Phyllodes tumours of the breast: the role of CD34, vascular endothelial growth factor and beta-catenin in histological grading and clinical outcome. Histopathology 2013;63:393-406.

27. Rungta S, Kleer CG. Metaplastic carcinomas of the breast: diagnostic challenges and new translational insights. Arch Pathol Lab Med 2012;136:896-900.

28. Kwon JE, Jung WH, Koo JS. The expression of metabolism-related proteins in phyllodes tumors. Tumour Biol 2013;34:115-24. 